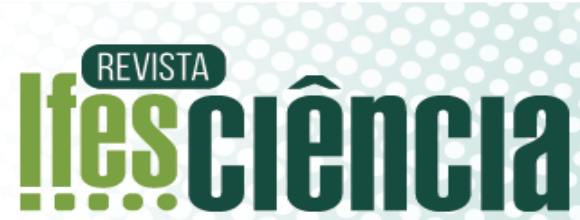

\section{ESTUDO POR MÉTODOS SEMI-EMPÍRICOS E DINÂMICA MOLECULAR DE POLÍMEROS CARREADORES DE ANTIBIÓTICOS}

\author{
STUDY BY SEMI-EMPIRICAL METHODS AND MOLECULAR \\ DYNAMICS OF ANTIBIOTIC CARRYING POLYMERS
}

${ }^{1}$ Arieli Santos de Andrade Segantine.

${ }^{2}$ Arlan da Silva Gonçalves.

\author{
${ }^{1}$ IFES Campus Vitória. E-mail: (arielisandrade@ gmail.com). \\ ${ }^{2}$ IFES Campus Vila Velha. E-mail: (agoncalves@ifes.edu.br). \\ *Autor de correspondência
}

Artigo submetido em 03/07/2020, aceito em 14/08/2020 e publicado em 28/08/2020.

Resumo: A presença de frações de antibióticos no meio ambiente tem gerado uma preocupação mundial. Maioria dos antibióticos não são totalmente absorvidos pelo organismo, sendo excretados através das fezes e urina dos seres humanos e animais. A contaminação do ambiente com antibióticos, principalmente no meio aquático, pode trazer diversos problemas à saúde, além do próprio ecossistema. Desta forma, foram utilizadas técnicas de modelagem molecular como a dinâmica molecular (DM) e o método semi-empírico PM7, para o estudo e predição computacional das interações inter e intra moleculares, em função do tempo entre os complexos Polietilenoglicol (PEG) / antibióticos e Polivinilpirrolidona (PVP) / antibióticos, sendo que tanto para o modelo do PEG quanto do PVP foram construídos hexâmeros e, no caso dos antibióticos, foram utilizados a Amoxicilina (AMX), Cefalexina (CFX), Azitromicina (AZT) e Penicilina (PEN), de modo a se verificar possível capacidade de adsorção no carreamento destes, quando presentes em água. Otimizações estruturais, além de cálculos estruturais e eletrônicos também foram executados, tanto para a extração de propriedades físico químicas, como também para o preparo dos arquivos entrada das simulações por DM. Foram simulados 8 sistemas: PEG-AMX, PEG-CFX, PEG-AZT, PEG-PEN, PVP-AMX, PVPCFX, PVP-AZT, PVP-PEN, centrado em uma caixa de simulação cúbica, solvatada com moléculas de água. As trajetórias das simulações por DM foram analisadas e os resultados mostraram que o sistema PVP-AZT se manteve mais estável, apresentando poucas variações conformacionais, além da própria estabilidade energética. O PVP foi o polímero que melhor interagiu com os antibióticos, sugerindo maior suscetibilidade de captura e carreamento, tendo como exceção o sistema PVP-PEN.

Palavras-chave: Modelagem molecular; dinâmica molecular; polímeros; antibióticos.

Abstract: The presence of fractions of antibiotics in the environment has generated worldwide concern. Most antibiotics are not fully absorbed by the body, being excreted through the feces and urine of humans and animals. Contamination of the environment with antibiotics, especially in the aquatic environment, can bring several health problems, in addition to the ecosystem itself. Thus, molecular modeling techniques such as molecular dynamics (DM) and the semi-empirical method PM7 were used for the study and computational prediction of inter and intra molecular interactions, as a function of the time between the Polyethylene Glycol (PEG) / antibiotics and Polyvinylpyrrolidone (PVP) / antibiotics complexes, and both for the PEG and PVP model hexamers were constructed and, 
in the case of antibiotics, Amoxicillin (AMX), Cephalexin (CFX), Azithromycin (AZT) and Penicillin (PEN) were used, in order to verify the possible ability to adsorption in their carrying, when present in water. Structural optimizations, in addition to structural and electronic calculations were also performed, both for the extraction of physical chemical properties, as well as for the preparation of the input files for the DM simulations. Eight systems were simulated: PEG-AMX, PEG-CFX, PEG-AZT, PEG-PEN, PVP-AMX, PVP-CFX, PVP-AZT, PVP-PEN, centered in a cubic simulation box, solvated with water molecules. The trajectories of the DM simulations were analyzed and the results showed that the PVP-AZT system remained more stable, presenting few conformational variations, besides the energy stability itself. PVP was the polymer that best interacted with antibiotics, suggesting greater susceptibility to capture and carry, with the exception of the PVP-PEN system.

Keywords: Molecular Modeling; Molecular dynamics; Polymers; Antibiotics.

\section{INTRODUÇÃO}

É impossível negar a importância da descoberta dos antibióticos no século $\mathrm{XX}$, além de ser considerada uma das conquistas científicas mais significativas daquele século, revolucionando a medicina (ARAÚJO, 2013). O desenvolvimento e uso de antibióticos na medicina humana e veterinária tem obtido resultados significativos na redução das taxas de mortalidade, morbidade social e epidemiológica, no tratamento de diversas doenças infecciosas (ALANIS, 2005). No entanto, os antibióticos foram reconhecidos recentemente como uma classe emergente de contaminantes ambientais. Há várias formas de vias de contaminação da água com antibióticos; eles podem chegar aos corpos hídricos através de escretas de homens e animais, disposição inadequada de resíduos de antibióticos, efluentes de Estações de Tratamento de Esgoto - ETE's (HOMEN, 2011).

Com as facilidades computacionais e avanços tecnológicos disponíveis, tornou-se possível simular o comportamento de moléculas in sílico com maior correspondência ao sistema real, analisando suas interações inter e intramoleculares e as reações químicas envolvidas, através de quebra e formação de ligações. Isso pode ser feito por meio do uso das técnicas de modelagem e dinâmica molecular (DM), onde o sistema é considerado como massas e molas, as cargas atômicas são pontuais e são utilizadas as equações do movimento de Newton (GONÇALVES, 2009).

Assim, dada a importância desta pesquisa, a proposta do trabalho consiste em realizar a simulação com os polímeros Polietilenoglicol e Polivinilpirrolidona, através da modelagem molecular, a fim de verificar as interações entre os polímeros e os antibióticos Amoxicilina, Cefalexina, Azitromicina e Penicilina e dessa forma verificar se algum desses polímeros obtiveram resultados que possibilite a ser utilizados como carreadores de algum desses antibióticos em água e, que possam vir, serem utilizados em tratamentos práticos.

\section{REFERENCIAL TEÓRICO}

\subsection{CONTAMINAÇÃO DE AMBIENTES AQUÁTICOS POR ANTIBIÓTICOS}

Os medicamentos têm um papel importante para saúde pública por serem utilizados para o combate de doenças de seres humanos e animais e, consequentemente, a prolongação de vida. Grande parte dos medicamentos utilizados pertencem a classe dos antibióticos. Segundo Araújo (2013) a presença destes, em mananciais, tem gerado uma série de estudos por serem persistentes no meio ambiente, ou seja, apresentarem a tendência de permanecerem no ecossistema pela resistência à degradação química e biológica, inclusive aos efeitos de processos microbianos. Além disso, são lipofílicos, bioacumulativos, 
biologicamente ativos, com elevada polaridade e natureza não volátil, o que dificulta sua total remoção através do tratamento de água, inclusive, água de consumo (TORRES, 2012).

Isto pode ser observado diante do número de publicações de revisão recentes sobre ocorrência, destino e ecotoxicidade de produtos farmacêuticos focados no ambiente aquático refletindo a importância emergente deste tópico. (BROWN et al, 2006, FARRÉ et al. 2008; GALÁN et al., 2008; KASSINOS; VASQUEZ; KÜMMERER, 2011; BOTTONI, CAROLI 2014; MATHON et al., 2016, AZANU et al, 2018, HE et al, 2019)

Uma vez que os sistemas atuais não conseguem remover todos os fármacos efetivamente, são necessárias melhorias e alterações para contornar este problema. A adsorção através de polímeros é um método que tem se mostrado eficaz e é comumente empregada para remoção de poluentes orgânicos na fase aquosa.

Assim, considerando o problema do uso indiscriminado de antibióticos no nosso país, além dos problemas ambientais e de saúde pública associado ao seu descarte inadequado, observou-se a demanda pela busca do desenvolvimento de uma tecnologia sustentável, direcionada para um tratamento que seja capaz de realizar remoção destes poluentes presentes na água.

\subsection{MODELAGEM MOLECULAR}

A modelagem molecular consiste em um conjunto de ferramentas para a construção, edição e visualização, análise e armazenamento de sistemas moleculares complexos. A computação tem se mostrado uma das técnicas mais importantes na química. A modelagem computacional usa modelos numéricos para explorar as estruturas e propriedades de moléculas individuais dos materiais (SHRIVER et al. 2008).

Os modelos moleculares computacionais são resultados das equações matemáticas que calculam as posições e as respostas dos elétrons e dos núcleos. Os modelos matemáticos são divididos em aproximações clássicas e quânticas. Os mecanismos clássicos olham moléculas como uma coleção de átomos e ligações que são tratados como "massas" e "molas". Informações como raios atômicos e rigidez das molas são usadas para encontrar as posições mais estáveis dos átomos. Os métodos quânticos utilizam aproximações numéricas da equação de Schrödinger, através de duas abordagens: semi-empírica e ab initio (SHARMA; GUPTA, 2009).

Nos métodos clássicos ou de mecânica molecular, o campo de forças é a função básica que os diferem, podendo ser totalmente empíricos, para definições de conformações de energias menores e assim mais estáveis, contrário dos métodos teóricos, passando pelos chamados métodos semi-empíricos, que apresentam truncamento na resolução numérica da equação de Schrödinger, onde são considerados apenas os elétrons da camada de valência, além de alguns parâmetros obtidos experimentalmente para calcular a energia e densidade eletrônica dos orbitais. A aplicação de um ou outro método depende do compromisso entre tempo e precisão dos resultados e da complexidade do sistema a ser analisado (BELLO, 2014).

\subsubsection{Método ab initio}

$\mathrm{O}$ método $a b$ initio é $a$ principal abordagem para resolver a equação de Schrödinger para moléculas poliatômicas de muitos elétrons. Ab initio significa, início, ou princípios fundamentais, implicando que os cálculos que utilizam esta abordagem requerem como parâmetros as constantes físicas universais que podem ser a velocidade da luz, constante de Planck, dentre outros (BELLO, 2014).

$\mathrm{O}$ método $a b$ inítio utiliza, como ponto de partida o método Hartree-Fock 
(HF) para soluções da equação de Schrödinger e são geralmente satisfatórios para uma variedade de comparações termodinâmicas e cinéticas. Este modelo aumenta em custo computacional de acordo com o aumento do tamanho da molécula. Isso faz com que eles não se tornem aplicáveis às moléculas com mais de 100 átomos (GONÇALVES, 2005).

\subsubsection{Métodos semi-empíricos}

Os métodos semi-empíricos são baseados no método Hartree-Fock e utilizam um parâmetro ajustado com dados experimentais. Também são conhecidos como orbitais moleculares e possuem os seguintes modelos: MNDO, AM1 e PM3, PM6, PM7 e RM1, que podem ser aplicados a moléculas compreendendo acima de 200 átomos pesados. Têm tido muito sucesso no cálculo da geometria de equilíbrio, incluindo as geometrias dos compostos que contém metais de transição e compostos organometálicos, além de um moderado sucesso para o cálculo das geometrias dos estados de transição. Eles, porém, não são satisfatórios para validações termodinâmicas e cinéticas ou para indicações conformacionais (GONÇALVES, 2005).

\subsubsection{Mecânica molecular}

A mecânica molecular considera a composição atômica da molécula interagindo entre si por forças harmônicas. Como resultado desta simplificação, a mecânica molecular é um método computacional relativamente rápido, que pode ser empregado no cálculo de estruturas moleculares pequenas e até de sistemas oligomoleculares (HÖLTJE et al., 2003).

Na mecânica molecular, os átomos são considerados como esferas unidos por molas de comprimento variável.

\subsubsection{Dinâmica molecular}

Dinâmica molecular é uma técnica de simulação computacional que permite predizer a evolução temporal das interações entre átomos e moléculas e estimar propriedades físicas e termodinâmicas relevantes para um determinado sistema. Simulação por dinâmica molecular constitui-se em um conjunto de condições iniciais (posições e velocidades iniciais de todas as partículas no sistema), interações potenciais para representar as forças entre todas as partículas e a evolução do sistema em função do tempo pela solução de um conjunto de equações de movimento para todas as partículas no sistema (ZHENG et al., 2008).

\subsection{ENERGIA DOS ORBITAIS HOMO E LUMO}

As energias dos orbitais de fronteira denominados HOMO (highest occupied molecular orbital) e LUMO (lowest unoccupied Molecular orbita), envolvem o princípio de que os elétrons em uma molécula não estão localizados entre átomos, mas em orbitais moleculares deslocalizados, no qual um orbital representa uma combinação linear dos orbitais atômicos. O orbitais HOMO e LUMO são propriedades importantes, pois, medem a capacidade doadora e receptora de elétrons, respectivamente. Assim quanto maior a energia do HOMO, maior será o caráter nucleofílico, ou seja, maior capacidade de doar elétrons e quanto menor for o nível energético do LUMO maior será o caráter eletrofílico, pois os elétrons estarão em órbita mais estável. (HONÓRIO; SILVA, 2011)

Dada a importância desta pesquisa, a proposta do trabalho tem o objetivo de Estudar, em nível molecular, através da técnica de Modelagem Molecular, como ocorrem as interações entre os antibióticos Amoxicilina, Cefalexina, Azitromicina e Penicilina e os polímeros Polietilenoglicol (PEG) e Polivinilpirrolidona (PVP), de modo que, em trabalhos posteriores, 
possam ser propostos melhores carreadores de antibióticos presentes em água, que possam vir, serem utilizados em tratamentos práticos.

\section{PROCESSOS METODOLÓGICOS /MATERIAIS E MÉTODOS}

As moléculas dos antibióticos e do hexâmero do PEG foram baixadas do banco de dados do PubChem (https://PubChem.ncbi.nlm.nih.gov/). Para o desenho tridimensional do hexâmero do polímero PVP e a otimização clássica das moléculas do hexâmero do polímero PEG, além dos antibióticos Amoxicilina, Cefalexina, Azitromicina e Penicilina, foi utilizado o programa GHEMICAL (ROWLE; HASSINEN, 2002), e o campo de forças TRIPOS 5.2. Utilizando $\mathrm{o}$ mesmo campo de forças, todas as moléculas de polímeros e antibióticos estudados, foram submetidas à análises conformacionais randômicas, de forma a se obter geometrias o mais próximo possível do mínimo global de energia clássica representada na figura 1 .

Figura 1: Geometrias otimizadas, a) Amoxicilina, b) Azitromicina c) Cefalexina,d) Penicilina, e) Hexâmero do PEG, f) Hexâmero do PVP
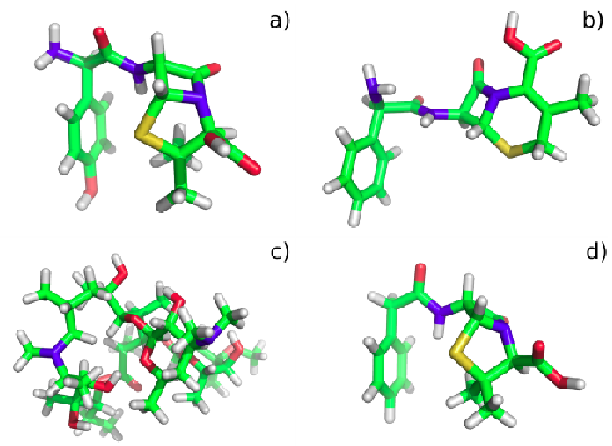

e)
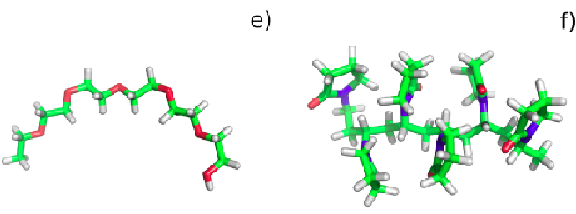

Fonte: Elaborado pelo autor (2019).

Com o auxílio do programa OPEN BABEL foi feita a conversão de arquivos no formato '. $g p r^{\prime}$ para o formato '.mop', para posteriormente, todas as moléculas individualmente foram otimizadas, utilizando o método semi-empírico PM7, implementado no MOPAC (MOPAC, 2016).

O Ajuste de cargas atômicas e preparo dos arquivos de topologias reconhecido pelo GROMACS, foram realizados usando o programa ACPYPE. Todavia, obter as configurações iniciais de moléculas não disponíveis em bancos de dados requer atribuição de cada parâmetro de ligação, interação e torção do campo de forças, procedimento esse muito trabalhoso. Assim foi utilizado os softwares ACPYPE (or AnteChamber PYthon Parser interfacE) (SILVA, 2012) e MKTOP (RIBEIRO; HORTA, e ALENCASTRO, 2012) que atribuem esses parâmetros de campo de forças como OPLS/AA e geram essas configurações em pouco tempo, detalhes sobre o passo à passo desta parametrização no Apêndice B. Com o ACPYPE, foi atribuído parâmetros de ângulos, diedros, constantes de força, disponível para vários métodos de cálculo de cargas parciais. Após a modelagem das moléculas, otimização e cálculos eletrônicos, foram realizadas as simulações por dinâmica molecular utilizando o pacote computacional GROMACS (ABRAHAM et al, 2014).

Para iniciar o processo de dinâmica Molecular foi criado o arquivo de coordenadas das estruturas e os arquivos de topologias, que contém parâmetros de ligações, ângulos, ângulos diedrais, esferas de van der Waals, cargas atômicas, constantes de forças, além de outros, de forma que seja reconhecido pelo GROMACS. Foram necessários também arquivos contendo os parâmetros da simulação, com extensão mdp, como número de passos, temperatura, pressão, além de outros, tanto para minimização de energia, como para as simulações por dinâmica molecular, onde é feita, inicialmente, uma equilibração do sistema 
de 500 ps, para relaxar o solvente em volta das estruturas dos antibióticos e polímeros e, posteriormente, as dinâmicas moleculares soltas.

Cada sistema foi composto por um hexâmero de polímero e uma molécula antibiótico, totalizando 8 sistemas de dinâmica, identificados da seguinte forma: PEG-AMX, PEG-CFX, PEG-AZT, PEGPEN, PVP-AMX, PVP-CFX, PVP-AZT, PVP-PEN, que foram centrados em caixas cúbicas, como mostrado na Figura 2, à qual foram inseridas moléculas de água e, em condições periódicas de contorno, para que sejam evitados os problemas de fronteira e, na temperatura de $310 \mathrm{~K}$ e pressão $1 \mathrm{~atm}$, de forma a se simular comportamentos mais próximos da realidade, sendo que, inicialmente cada antibiótico foi colocado há uma distância média de $20 \AA$ do polímero, utilizando o PYMOL, para o acompanhamento de sua a evolução temporal e verificar suas interações.

Figura 2: Caixa cúbica do sistema PVP-AZT solvatada

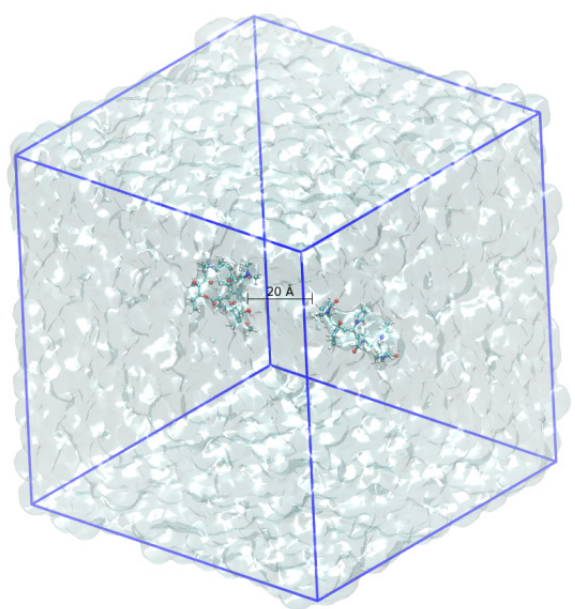

Fonte: Elaborado pelo autor (2019)

O campo de forças utilizado para a parametrização dos hexâmeros dos polímeros e dos antibióticos, foi $\mathrm{o}$ OPLS/AA e o modelo de água adotado, foi o TIP3P (ABRAHAM et al, 2014).

Após a imersão de todos os sistemas minimizados foram, então, submetidos à simulações por dinâmica molecular em 500 ps de dinâmica com restrição de posição para acomodação das moléculas de água no sistema, isso com condições periódicas de contorno, após este procedimento, foram executadas 8 simulações de dinâmica molecular de $20 \mathrm{~ns}$ na temperatura de $310 \mathrm{~K}$ e 1 atm, dependendo da evolução temporal da estabilização das interações entre os polímeros e os antibióticos. Em seguida, após a escrita dos arquivos das trajetórias (.trr e .xtc) e dos arquivos das energias (.edr), os resultados foram analisados, discutidos e concluídos.

As trajetórias das simulações por dinâmica molecular foram analisadas pelo desvio da raiz média quadrática (RMSD), das estrutura dos antibióticos e polímeros de cada sistema simulado, ligações hidrogênio, energia de van der Walls e momento dipolo, utilizando programas específicos que fazem parte do pacote GROMACS.

\section{RESULTADOS E DISCUSSÃO}

Os resultados dos cálculos eletrônicos, estão descritos na tabela 1.

Tabela 1: Resultados das propriedades estruturais eletrônicas dos antibióticos e polímeros em estudo

\begin{tabular}{|c|c|c|c|c|c|c|}
\hline Estruturas & $\begin{array}{c}\Delta h_{f_{f} /} \\
\text { Kcal.mol }^{-1}\end{array}$ & $\begin{array}{c}\text { Energia total / } \\
\mathrm{Ev}\end{array}$ & $\begin{array}{c}\text { Dipolo / } \\
\text { D }\end{array}$ & $\begin{array}{l}\text { eHomo/ } \\
\text { e.V }\end{array}$ & $\begin{array}{l}\text { ELumo/ } \\
\text { e.V }\end{array}$ & Ebg / e.V \\
\hline Amoxicilina & $-693,24$ & $-4433,95$ & 4,87 & $-9,21$ & $-0,71$ & 8,50 \\
\hline Cefalexina & $-385,30$ & $-4110,57$ & 5,19 & $-9,45$ & $-0,94$ & 8,51 \\
\hline Penicilina & $-10,30$ & $-2548,77$ & 4,67 & $-8,90$ & $-0,78$ & 8,12 \\
\hline Azitromicina & $-2665,71$ & $-9557,97$ & 6,12 & $-8,52$ & 1,12 & 9,65 \\
\hline PEG & $-975,61$ & $-3588,79$ & 0,69 & $-10,04$ & 0,73 & 10,77 \\
\hline PVP & $-1384,56$ & $-8071,19$ & 11,50 & $-8,85$ & 1,12 & 9,97 \\
\hline
\end{tabular}

Fonte: Elaborado pelo autor (2019)

$\Delta \mathrm{H}_{\mathrm{f}}=$ calor da entalpia de formação

$\mathrm{E}_{\mathrm{bg}}=$ Energia de band gap, ou gap energético.

Assim, é possível observar que o calor de formação obtidos das moléculas estudadas obtidos, tal grandeza refere-se à quantidade de energia liberada ou absorvida na síntese de uma dada substância a partir de seus elementos, de 
modo que as moléculas com menor calor de formação são esperados serem mais estáveis. Toda as moléculas se apresentaram estáveis, porém com variações expressivas em seus valores, com a penicilina que apresentou maior valor de $-10,30 \mathrm{Kcal}^{\mathrm{mol}}{ }^{-1}$ e a Azitromicina que apresentou menor valor de $-2665,71$ Kcal.mol ${ }^{-1}$. Os resultados de energia total negativos, mostra que as moléculas estão estáveis.

Por meio dos valores de momento dipolo verificou-se que todas as moléculas estudadas são polares. Dentre os antibióticos os valores não sofreram variações muito expressivas, porém entre os polímeros é observada crescimento significativo do menor valor para o PEG $(0,69 \mathrm{D})$ para o maior o PVP $(11,50 \mathrm{D})$. Quanto aos orbitais moleculares HOMO e LUMO, indicam possíveis tendências de uma determinada reação química ocorrer, através do conceito de nucleofilicidade e eletrofilicidade.

Uma análise do HOMO fornece resultados sobre a tendência de um composto possuir um maior caráter nucleofílico enquanto LUMO eletrofílico, ou seja, no caso de uma reação $\mathrm{SN}^{2}$ onde se tem um grupo epóxi atacando um cloreto de alquila, pelo fato do epóxi possuir um orbital HOMO localizado e pronunciado no oxigênio, será o nucleófilo. Por outro lado, o cloreto de alquila, por possuir o orbital LUMO localizado, predominantemente, no carbono, será o eletrófilo.

$\mathrm{O}$ valor do GAP (módulo da diferença das energias dos orbitais HOMO e LUMO): |(عLUMO - عHOMO)| é um parâmetro utilizado para definir a estabilidade molecular, considerando que um valor alto sugere alta estabilidade e baixa reatividade química; um valor baixo sugere o contrário. Sendo assim, a estabilidade da molécula é diretamente proporcional ao valor de GAP.

Nota-se que as energias do HOMO e LUMO para a molécula menos estável foram de -8,89 eV e -0,78 eV (Penicilina), e para a molécula mais estável foram $10,03 \mathrm{eV}$ e $0,730 \mathrm{eV}$ (PEG), onde os valores de GAP para essas moléculas são $8,11 \mathrm{eV}$ e $10,76 \mathrm{eV}$, respectivamente.

\subsection{ANÁLISE DOS RESULTADOS DAS SIMULAÇÕES POR DINÂMICA MOLECULAR}

\subsubsection{Análise dos Gráficos de RMSD}

O RMSD ou Desvio da Raiz Média Quadrática (DRMQ), é o cálculo feito para comparação entre estruturas e, no caso dos resultados de $\mathrm{DM}$, pode ser estendido à comparação entre diversas conformações espaciais assumidas pelo sistema ao longo do tempo em relação à estrutura média temporal deste conjunto.

A análise do RMSD representa a variação da posição das moléculas do polímero e do antibiótico ao longo da dinâmica, em relação à sua posição no tempo da simulação. Com o RMSD podemos avaliar durante a trajetória de simulação, a oscilação do sistema, pequenas oscilações de RMSD correspondem a posições de equilíbrio do sistema, enquanto mudanças bruscas na média denotam mudanças importantes na conformação de determinada molécula.

Figura 3: Gráfico de RMSD para os sistemas: PEG-AMX (preto), PEG-CFX (vermelho), PEG-AZT (verde) e PEG-PEN (azul)

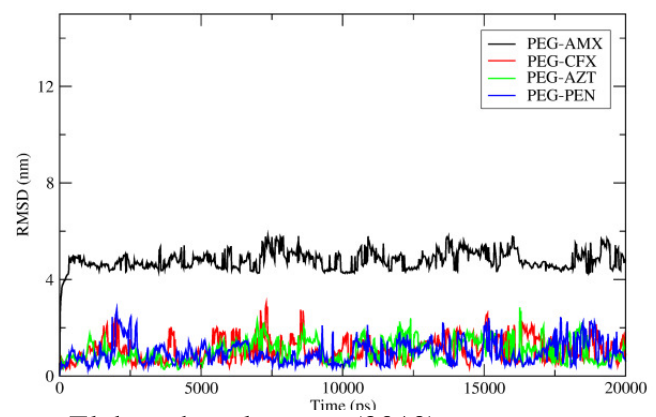

Fonte: Elaborado pelo autor (2019) 
Figura 4: Gráfico de RMSD. Sistema PVPAMX (preto). Sistema PVP-CFX (vermelho). Sistema PVP-AZT (verde). Sistema PVP -PEN (azul)

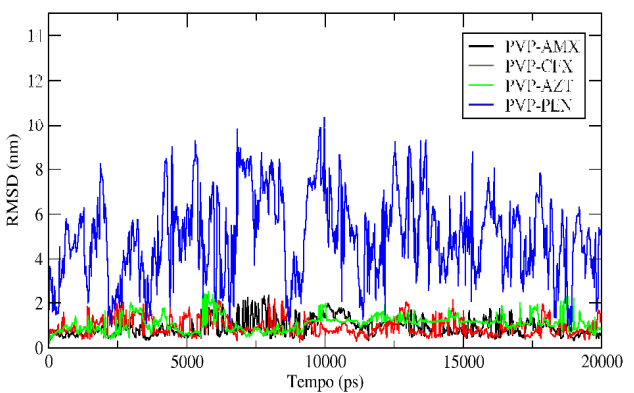

Fonte: Elaborado pelo autor (2019).

Quando analisado o perfil de comparação do RMSD na figura 3, entre o PEG e os antibióticos observou-se que os sistemas PEG-CFX, PEG-AZT,PEGAMX, e PEG-PEN, se mantiveram em uma baixa flutuação demostrando maior estabilidade. Em relação ao PVP (figura 4), os sistemas PVP-CFX, PVP-AZT e PVP-AMX, sofreram oscilações menores e se mantiveram com uma flutuação baixa e mais estáveis, com comportamento mais uniforme, e no sistema PVP-PEN fica evidenciado o comportamento com maiores oscilações em relação aos demais sistemas e apresentou maiores deformações estruturais em sua estrutura durante a simulação, com grandes picos e variação em sua conformação.

Figura 5: Gráfico com as médias e seus respectivos desvios padrões para os sistemas: PEG-AMX,

PEG-CFX, PEG-AZT, PEG-PEN

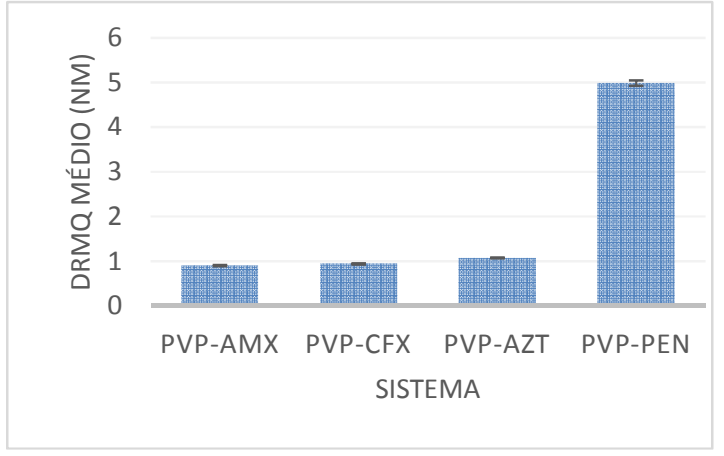

Figura 6: Gráfico com as médias e seus respectivos desvios padrões para os sistemas: PVP-AMX, PVP-CFX, PVPAZT, PVP-PEN

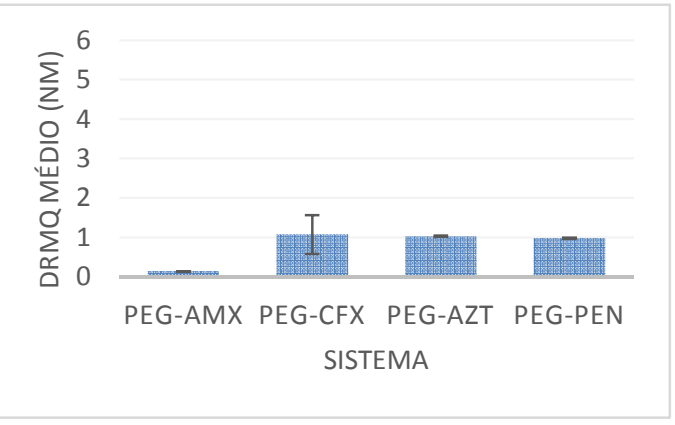

Fonte: Elaborado pelo autor (2019).

Para se ter uma análise mais qualitativa, foram extraídas as médias e os desvios padrões do RMSD, e organizados em 2 gráficos nas Figuras 5 e 6, onde foi computada sobre os registros feitos no intervalo de tempo de $20 \mathrm{~ns}$ de simulações.

Para cada sistema, foi medida as médias de desvio para os antibióticos e polímeros, a partir destas análise, nota-se que as simulações entre os antibióticos e o PEG apresentaram menores valores em sua média de flutuação das moléculas, indicando assim, a estabilidade na dinâmica das simulações, destacando o sistema PEG-AMX com menor valor em relação a todos os sistemas. Nas análises dos antibióticos com o PVP os sistemas PVP-AMX, PVP-CFX e o PVP-AZT, também mantiveram valores próximos de 1 ns na média, indicando também uma estabilidade na dinâmica. Em relação ao sistema PVP-PEN, verificou-se um comportamento mais atípico quando comparado aos outros sete sistemas, ao se observar que o desvio médio foi perceptivelmente acima dos valores observados para os demais sistemas, indicando maior flutuação da molécula de 
$5 \mathrm{~nm}$, corroborando com os resultados apresentados nos gráficos.

\subsubsection{Análise dos gráficos das distâncias entre os centros de massa}

As figuras 7-10 representam as distâncias entre os centros de massa das moléculas dos hexâmeros de polímeros e os antibióticos no tempo da simulação.

Figura 7: Distâncias entre o PEG e o AMX (preto) e PVP e o AMX (vermelho) ao longo da simulação

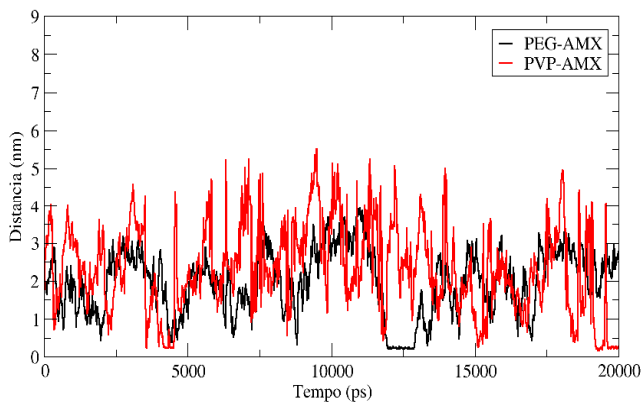

Fonte: Elaborado pelo autor (2019).

Figura 8 - Distâncias entre o PEG e o CFX (preto) e PVP e o CFX (vermelho) ao longo da simulação

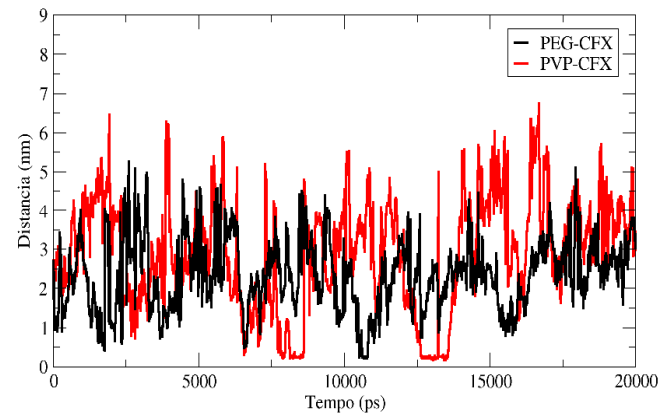

Fonte: Elaborado pelo autor (2019).

Figura 9 - Distâncias entre o PEG e o AZT (preto) e PVP e o AZT (vermelho) ao longo da simulação

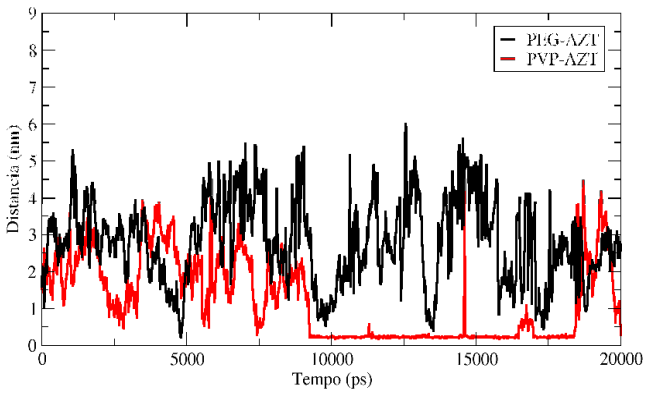

Fonte: Elaborado pelo autor (2019).

Figura 10: Distâncias entre o PEG e o PEN (preto) e PVP e o PEN (vermelho) ao longo da simulação

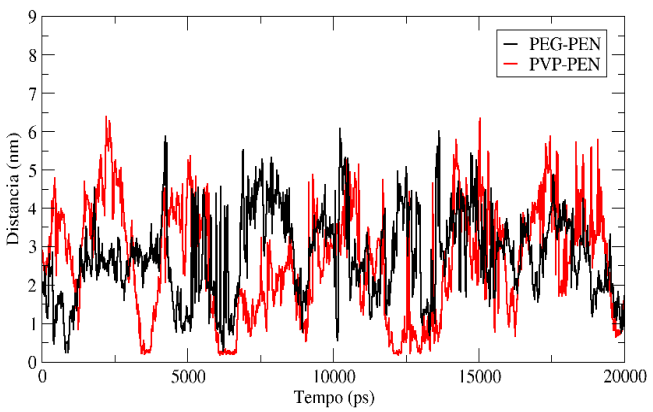

Fonte: Elaborado pelo autor (2019).

A partir da análise das distâncias entre os centros de massa da Figura 7, verifica-se que para a simulação entre o PEG-AMX houve grande variação entre as distâncias, através do gráfico é possível observar que as distâncias ficam oscilando em torno de 0 e $4 \mathrm{~nm}$ durante quase toda a simulação com um período de aproximação no tempo de 12000 ps. Entre o PVP-AMX as distâncias apresentaram muitas oscilações com alguns picos de aproximações que oscilaram de 0 a 5,6 nm. Nas simulações da Figura 8 entre o PEGCFX, o sistema apresentou uma trajetória bastante oscilante e distâncias variando de 0 a $6 \mathrm{~nm}$. O sistema de simulação entre o PVP-CFX apresentou variações nas distâncias com valores superiores em relação ao PEG, mas, foi possível observar pequenos períodos de aproximação no tempo de aproximadamente 7800 ps e 12500 ps. Analisando os gráficos da dinâmica na Figura 9 do PVP-AZT observa-se um resultado interessante, uma estabilidade e redução da distância por um tempo longo em torno de 9000 ps a aproximadamente 14000 ps, sugerindo uma maior interação entre as moléculas, é importante observar que dentre as moléculas de antibióticos, a azitromicina é a única que não tem o enxofre em sua estrutura, o pode ter levado a uma melhor interação com o PVP. Posteriormente elas se afastam rapidamente a $4,5 \mathrm{~nm}$ e voltam 
a aproximar-se novamente. Em relação ao sistema de simulação do PEG-AZT houve muita variação nos valores das distâncias em relação ao tempo variando de $0,5 \mathrm{~nm}$ a $6 \mathrm{~nm}$, mantendo-se na maior parte do tempo distante. Na simulação dos sistemas da Figura 10 do PEG-PEN as distâncias também oscilaram entre 0 e $6 \mathrm{~nm}$ durante toda simulação não havendo aproximações significativas e para o PVP-PEN os valores de distância também oscilaram de 0 a $6,5 \mathrm{~nm}$, havendo momentos de aproximação nos tempos de 3300 ps, 6200 ps e 11700 ps, também pouco significativas.

Tabela 2: Média e desvio padrão das distâncias para os sistemas de simulação em: PEG-AMX, PEG-CFX, PEG-AZT, PEG-PEN

\begin{tabular}{lcccc}
\hline & \multicolumn{5}{c}{ SISTEMAS POLÍMEROS E ANTIBIÓTICOS } \\
& PEG-AMX & PEG-CFX & PEG-AZT & PEG-PEN \\
\hline Distância Média (nm) & 2,34 & 2,89 & 2,8 & 2,64 \\
Desvio Padrão & 1,2 & 1,44 & 1,15 & 1,47 \\
\hline
\end{tabular}

Fonte: Elaborado pelo autor (2019).

Tabela 3 : Média e desvio padrão das distâncias para os sistemas de simulação em: PVP-AMX, PVP-CFX, PVP-AZT, PVP-PEN.

\begin{tabular}{lcccc}
\hline & \multicolumn{4}{c}{ SISTEMAS POLÍMEROS E ANTIBIÓTICOS } \\
& PVP-AMX & PVP-CFX & PVP-AZT & PVP-PEN \\
\hline Distância Média (nm) & 1,98 & 2,33 & 1,23 & 2,76 \\
Desvio Padrão & 0,86 & 0,94 & 1,09 & 1,15 \\
\hline
\end{tabular}

Fonte: Elaborado pelo autor (2019).

Para se ter uma análise mais clara, foram extraídas as médias e os desvios padrões das distâncias, e organizados nas tabelas 2 e 3 , contendo todos os complexos estudados. A partir destas análises, nota-se que na média, os sistemas PEG-CFX e PEG-AZT, foram os que se mantiveram mais distantes e o PEG-AMX o que se manteve mais próximo. Em relação aos sistemas com o hexâmero do PVP o PVP-
AZT foi o que apresentou menor distância, e o PVP-CFX maior distância. Dentre os dois polímeros foi observado que o PVP, se manteve em menor distância entre os antibióticos e com menores desvios padrão, apenas a penicilina apresentou maior distância.

\subsubsection{Análise dos gráficos de energias}

Foram realizadas análises de interações energéticas de dois tipos: a energia eletrostática e a energia de van der Waals dos sistemas calculado no GROMACS, afim de, analisar no decorrer de sua trajetória se o sistema atingiu o equilíbrio.

\subsubsection{Energia de van der Waals}

Quando o meio permite o estabelecimento simultâneo de um número significativo de interações de van der Waals, essas forças somadas exercem importante papel no processo de reconhecimento molecular e estabilização de complexos ligante- receptor. Neste trabalho foram descritas pelo potencial de Lennard-Jones de curto alcance.

Figura 11: Simulação de energia de van der Waals para os sistemas estudados:

PEG-AMX (preto) e PVP-AMX (vermelho)

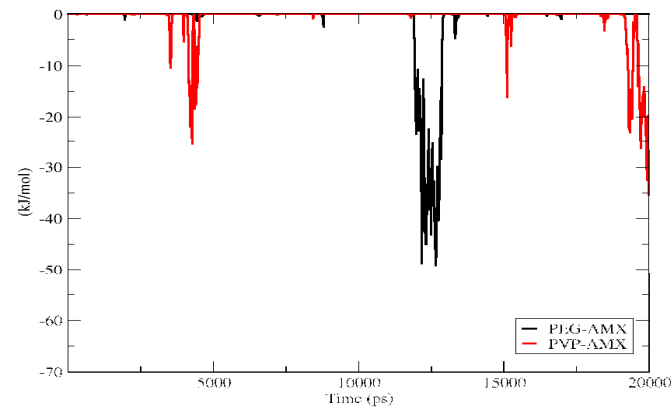

Fonte: Elaborado pelo autor (2019).

Figura 12: Simulação para energia de van der Waals para os sitemas estudados: PEGCFX (preto) e PVP-CFX (vermelho) 


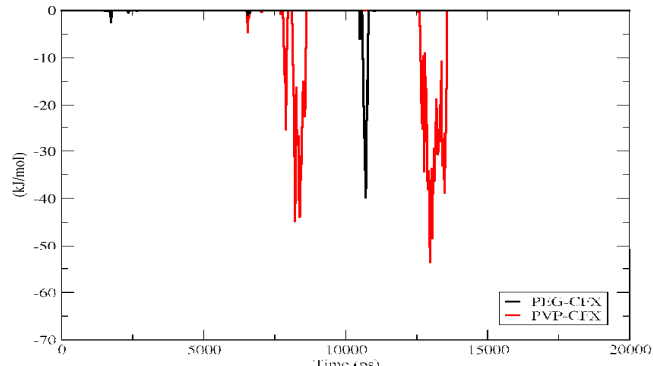

Fonte: Elaborado pelo autor (2019).

Figura 13: Simulações de energia de van der Waals, para os sistemas estudados: PEG-AZT (preto) e PVP-AZT (vermelho)

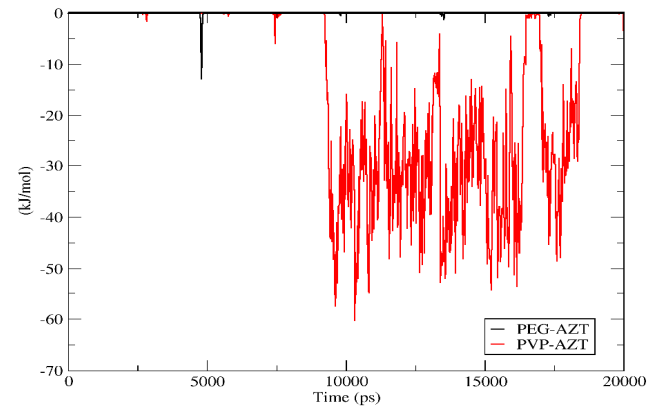

Fonte: Elaborado pelo autor (2019).

Figura 14: Simulações de energia de van der Waals para os sistemas: PEG-PEN (preto) e PVP-PEN (vermelho)

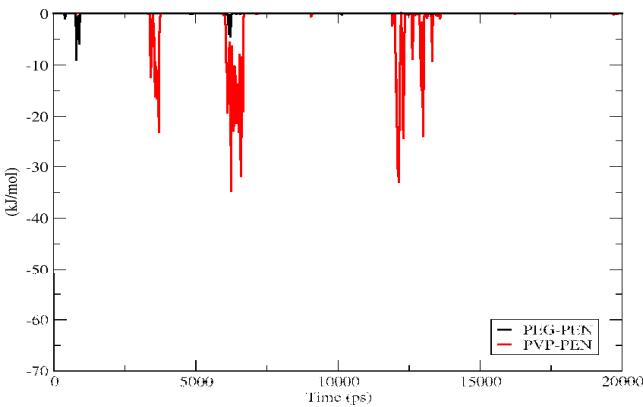

Fonte: Elaborado pelo autor (2019).

As interações de Leonnard-Jones (LJ) diminuem rapidamente com o aumento da distância, portanto ao analisar os gráficos da Figura 11 de trajetória das simulações das moléculas de polímeros e dos antibióticos observou-se que nos sistemas PEG-AMX e PVP-AMX houve períodos de interações mais forte entre as moléculas no tempo de 3000 a 4000 ps, 15000 ps e entre 18000 a 20000 ps. Nos sistemas PEG-CFX da Figura 12 houve poucas interações e com menores intensidades com valores de $-40 \mathrm{~kJ} / \mathrm{mol}$ no tempo de 10000 ps, sistema PVP-CFX, apresentou maiores períodos de interações com maiores intensidades com saltos consideráveis nos tempos de 6000 ps e $13000 \mathrm{ps}$ chegando a $-55 \mathrm{~kJ} / \mathrm{mol}$. Entre os sistemas PVP-AZT figura 13, observou-se interações de pequenas intensidades demostrando um distanciamento maior entre as moléculas, já as moléculas do PEG-ATZ foram quem demostraram maiores interações energéticas tanto no tempo quanto na intensidade no período entre 9000 ps a 19000 ps mostrando uma interação mais forte. Os sistemas PEGPEN também observou-se poucas interações e baixas intensidades e o PVPPEN maiores períodos de interações, ou seja, quanto mais forte as interações energéticas observadas, menores as distância entre as moléculas do polímero e antibióticos durante as simulações.

\subsubsection{Energia eletrostática (COULOMB)}

O valor da energia coulômbica entre os polímeros e antibióticos plotadas nos gráficos das Figuras 15, 16, 17 e 18 foi menor em relação a energia de van der Waals. A curva que representa o sistema PEG-AMX não apresentou grande flutuação dos valores durante a simulação. O PVP-AMX também se manteve com pouca flutuação com um pico de queda na energia em 19000 ps. O sistema PEGCFX, também não apresentou alteração significativa durante a simulação. No sistema PVP-CFX houve dois picos de queda na energia no tempo de 7500 ps e um pico de queda brusca em 13000 ps retornando para zero. No sistema PVPAZT manteve-se constante e bem próximo de zero durante quase toda simulação. No 
entanto o sistema PEG-AZT apresentou uma flutuação de energia em 9000 ps, mantendo-se até 19000 ps, indicando interação mais forte da molécula do polímero e o antibiótico neste intervalo. No sistema PEG-PEN a curva de energia eletrostática não apresentou grandes oscilações, mantendo-se próximo de zero durante toda a simulação. O sistema PVPPEN apresentou 3 períodos curtos de interação em 4000 ps 6000 ps e 12000 ps.

A energia de van der Waals, calculada com um potencial de LennardJones apresentou valores menores de energia para os sistemas, demonstrando uma menor distância nos períodos de interação mais forte entre as moléculas dos polímeros com os antibióticos.

Figura 15: Energia coulômbica para os sistemas PEG-AMX (preto) e PVP-AMX (vermelho)

Fonte: Elaborado pelo autor (2019).

Figura 16: Energia coulômbica para os

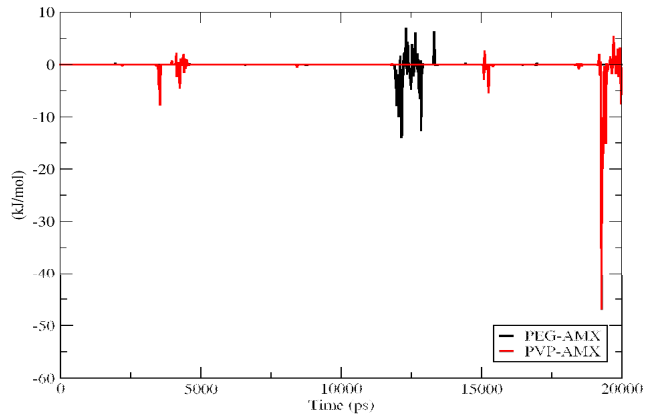

sistemas PEG-CFX (preto) e PVP-CFX (vermelho)

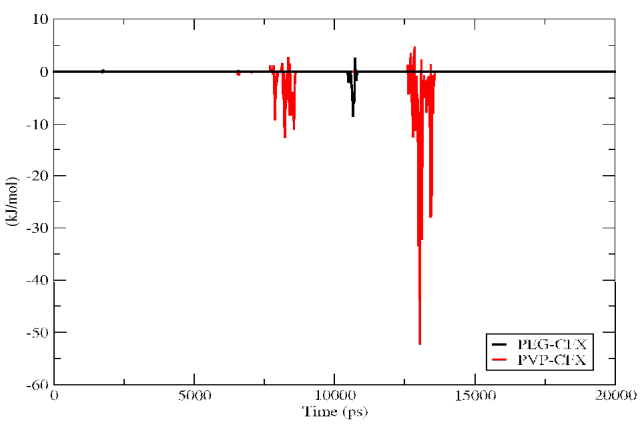

Fonte: Elaborado pelo autor (2019).

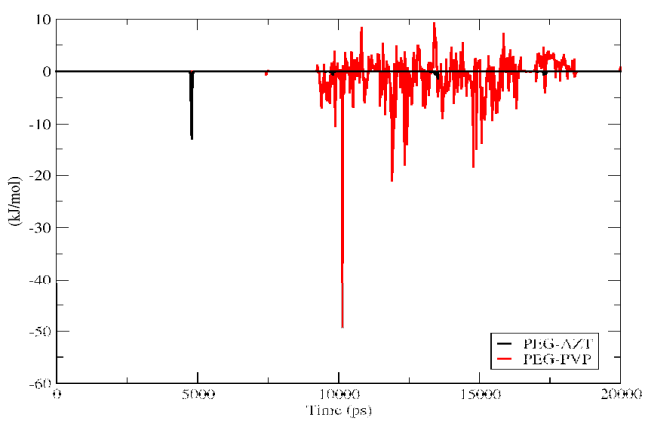

Figura 17: Energia coulômbica para os sistemas PEG-AZT (preto) e PVP-AZT (vermelho)

Fonte: Elaborado pelo autor (2019).

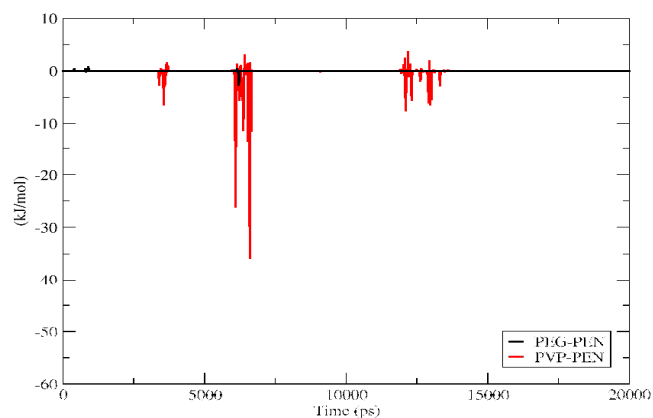

Figura 18: Energia coulômbica para os sistemas PEG-PEN (preto) e PVP-PEN (vermelho)

Fonte: Elaborado pelo autor (2019)

\subsubsection{Análise dos gráficos das ligações hidrogênio}

A DM também permite acompanhar as interações do tipo ligação hidrogênio ao longo do tempo. As interações por ligação hidrogênio entre antibióticos e polímeros são fatores importantes para avaliar a estabilização da estrutura. Durante a trajetória das simulações de Dinâmica molecular, foi efetuada uma análise da formação de ligação hidrogênio entre os polímeros e os antibióticos mostrados nas figuras 19 a 22. 
Figura 19: Distribuição das ligações hidrogênio dos sistemas: PEG-AMX (preto) e PVP-AMX (vermelho)

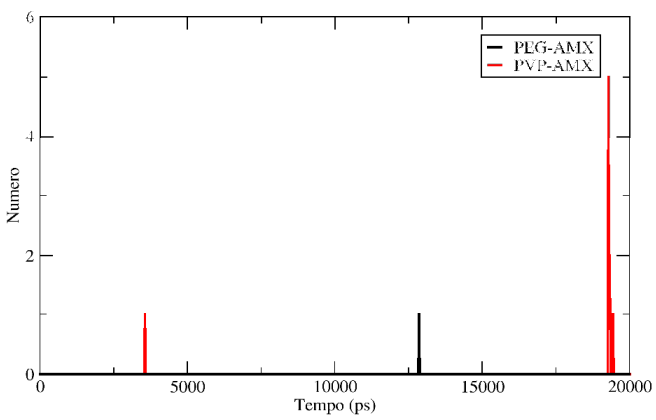

Fonte: Elaborado pelo autor (2019)

Figura 20: Distribuição das ligações hidrogênio dos sistemas: PEG-CFX (preto) e PVP-CFX (vermelho)

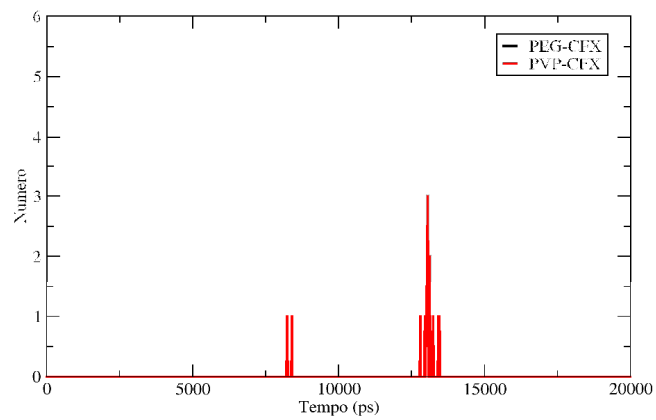

Fonte: Elaborado pelo autor (2019).

Figura 21: Distribuição das ligações hidrogênio dos sistemas: PEG-AZT (preto) e PVP-AZT (vermelho)

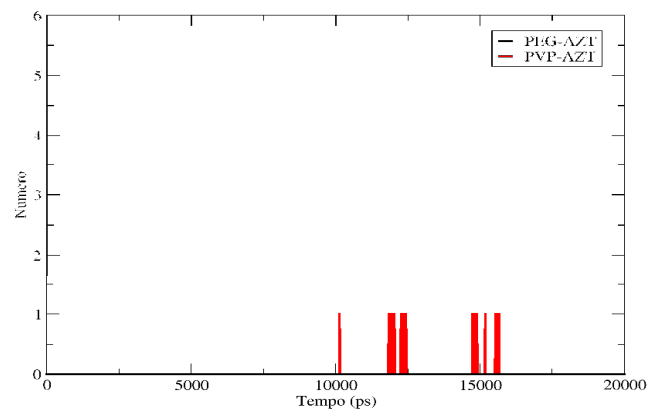

Fonte: Elaborado pelo autor (2019).
Figura 22: Distribuição das ligações hidrogênio dos sistemas: PEG-PEN (preto) e PVP-PEN (vermelho)

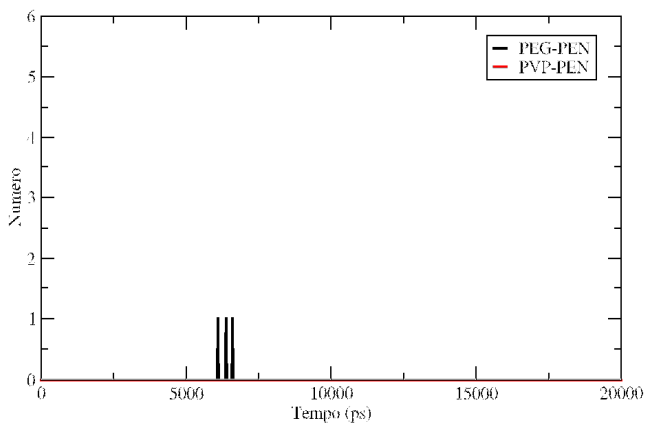

Fonte: Elaborado pelo autor (2019).

A distribuição das ligações hidrogênio do sistema com tempo de simulação de 20000 ps foi realizada somente entre a interação do polímero e o antibiótico, os resultados foram dispostos em gráficos, onde os picos representam o número de ligações hidrogênios em determinado tempo. Observou-se que houve 1 ligação hidrogênio no sistema AMX-PVP no tempo de aproximadamente 12700 ps e 8 ligações na simulação do sistema PEG-AMX no tempo de 3000 ps e aproximadamente 19000 ps. Nas simulações do sistema PVP-CFX ocorreu 2 ligação no período de 3000 ps e 12 ligações no intervalo de 12800 ps a 13400 ps. Nos sistema PVP-AZT houve 1, 3 e 3 ligações no tempo de 10140 ps, 11900 ps e 14700 ps respectivamente. No sistema de simulação entre o PEN-PVP, também não foi possível observar as ligações no período simulados, e no sistema PEN-PEG, ocorreram 3 ligações no tempo entre 6100 e 6600 ps respectivamente.

\subsubsection{Análise dos gráficos do momento dipolo}

O momento dipolo representa a intensidade do dipolo elétrico e medem a polaridade do sistema. Nas Figuras 23, 24, 25 e 26 pode-se observar a variação das curvas de momento dipolo da trajetória das simulações dos polímeros e antibióticos. 
Figura 23: Evolução temporal do momento dipolo para os sistemas de simulação:

PEG-AMX (preto) e PVP-AMX (vermelho)

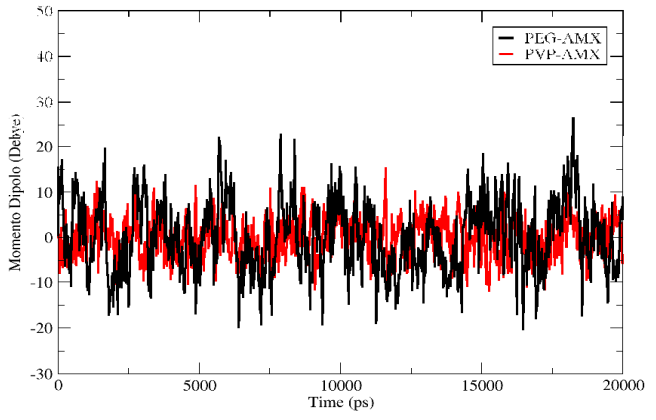

Fonte: Elaborado pelo autor (2019).

Figura 24: Evolução temporal do momento dipolo para os sistemas de simulação: PEG-CFX (preto) e PVP-CFX (vermelho)

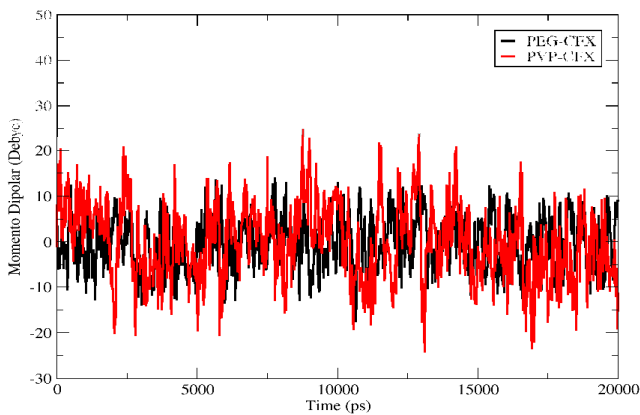

Fonte: Elaborado pelo autor (2019).

Figura 25: Evolução temporal do momento dipolo para os sistemas de simulação: PEG-AZT (preto) e PVP-AZT (vermelho)

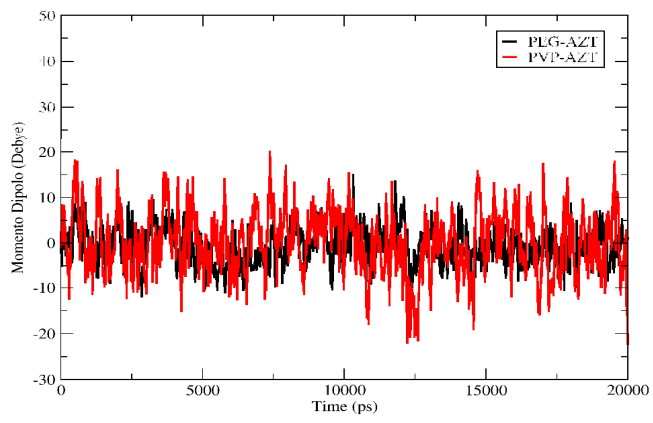

Fonte: Elaborado pelo autor (2019).
Figura 26: Evolução temporal do momento dipolo para os sistemas de simulação: PEG-PEN (preto) e PVP-PEN (vermelho)

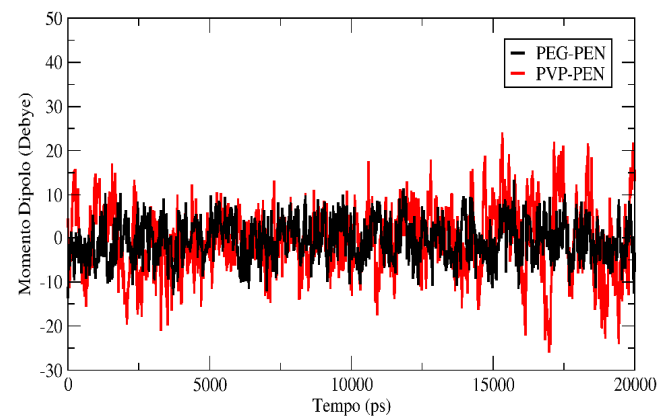

Fonte: Elaborado pelo autor (2019).

Entre o PEG-AMX, a variação do momento dipolo se manteve entre -20 debye e 20 debye, em constante oscilação entre o maior e menor valor durante toda simulação. O sistema PVP-AMX teve uma menor variação de seus valores se mantendo entre -10 e 15 debye e com muita oscilação. Em relação ao sistema PEG-CFX se manteve em -10 e 12 debye com uma oscilação mais contínua e equilibrada, enquanto que para o PVPCFX os valores se alternaram entre -25 e 25 debye, com muitas oscilações. Entre as interações PEG-AZT, os valores se apresentaram entre -10 e 15 debye, com poucas oscilações e um movimento mais estável. Entre o PVP-AZT, os valores variaram entre -20 e 20 debye, mas, com poucos picos de variação. $\mathrm{Na}$ trajetória do sistemas PEG-PEN o momento dipolo oscilou muito pouco entre -10 e 10 debye de forma constante e equilibrada. $\mathrm{O}$ sistema PVP-PEN, apresentou poucas oscilações até aproximadamente 15000 ps, passando a ter maiores picos aumentando e diminuindo seus valores até 20000 ps.

Tabela 4: Média e desvio padrão do momento dipolo para os sistemas de simulação em debye: PEG-AMX, PEGCFX, PEG-AZT, PEG-PEN 


\begin{tabular}{lcccc}
\hline & \multicolumn{5}{c}{ SISTEMAS POLÍMEROS E ANTIBIÓTICOS } \\
& PEG-AMX & PEG-CFX PEG-AZT & PEG-PEN \\
\hline Momento dipolo (Médio) & $-0,28 \mathrm{D}$ & $-0,41 \mathrm{D}$ & $-0,81 \mathrm{D}$ & $0,65 \mathrm{D}$ \\
Desvio Padrão & 4,62 & 5,79 & 4,13 & 4,85 \\
\hline
\end{tabular}

Fonte: Elaborado pelo autor (2019)

Tabela 5: Média e desvio padrão do momento dipolo para os sistemas de simulação em debye: PVP-AMX, PVPCFX, PVP-AZT, PVP-PEN

\begin{tabular}{lcccc}
\hline & \multicolumn{4}{c}{ SISTEMAS POLÍMEROS E ANTIBIÓTICOS } \\
& PVP-AMX & PVP-CFX & PVP-AZT & PVP-PEN \\
\hline Momento dipolo (Médio) & $0,14 \mathrm{D}$ & $-0,14 \mathrm{D}$ & $0,08 \mathrm{D}$ & $-0,53 \mathrm{D}$ \\
Desvio Padrão & 8,01 & 8,48 & 7,15 & 7,98 \\
\hline
\end{tabular}

Fonte: Elaborado pelo autor (2019).

Avaliação do momento dipolo durante a simulação dos sistemas podem ser observados nas tabelas 6 e 7. Quanto ao polímero PEG o melhor resultado apresentado foi do AMX com uma média de $-0,28$ de momento dipolo. Em relação as simulações com o polímero PVP, o antibiótico que apresentou o melhor resultado para o momento dipolo foi $o$ AZT com uma média de 0,08 corroborando os resultados das análises anteriores.

\section{CONCLUSÕES}

A proposta do presente estudo foi a realização de simulações de dinâmica molecular entre os antibióticos amoxicilina, cefalexina, azitromicina e penicilina e polímeros polietilenoglicol e o polivinilpirrolidona no sentido de verificar a possibilidade de captura de tais antibióticos por meio carreamento por adsorção em meio aquoso.

As simulações foram realizadas em 8 sistemas diferentes com os pares PEGAMX, PVPAMX, PEG-CFX, PVP-CFX, PEG-AZT, PVP-AZT, PEG-PEN E PVPPEN.

A julgar pelos resultados de RMSD, os sistemas e moléculas foram construídos adequadamente. O sistema que se apresentou em maior equilíbrio e menores variações de conformação das moléculas foi o PVP-AZT, porém cabe destacar o caso do sistema PVP-PEN, que apresentou as maiores flutuações.

- As análises de ligações hidrogênio das simulações do sistema apresentaram poucas ou nenhuma ligação intermolecular entre os polímeros e antibióticos. O PVP foi o polímero que realizou mais ligações de hidrogênio com o AMX, CFX e AZT, o que se deve ao fato do PVP conter grupos elétrons doadores como nitrogênio e oxigênio.

A análise de área acessível ao solvente mostrou que assim como houve variações nas conformações nas análises de RMSD, isso também aconteceu nesta propriedade.

Quanto a distância do centro de massa, as simulações mostraram que, entre polímeros e antibióticos não foi possível obter para todos os sistemas os resultados esperado e propostos por esta pesquisa, a não ser pelo sistema PVP-AZT, que apresentou um longo período de interação durantes as simulações, resultados estes, que podem ser percebidos e observados também nas análises de interações energéticas eletrostática e de van der Waals.

\section{REFERÊNCIAS}

ABRAHAM, M. J. et al. GROMACS user manual version 5.0.5. Departamento f Biophysical Chemestry, university of Groninger e Royal Institute of Technology and Uppsala University.Suécia, 2015. Disponível em: www.gromacs.org. Acesso em: 20. ago. 2017.

ALANIS, J. A. Resistance to Antibiotics: Are We in the Post-Antibiotic Era? Archives of. Medical Reserch, Uppsala, v. 36, p. 697-705, 2005. 
ARAÚJO, A. B. N. As doenças infeciosas e a História dos Antibióticos. 2013. 79f. Dissertação (Mestrado em Ciências Farmacêuticas) - Universidade Fernando Pessoa, Porto, 2013.

AZANU, D. et al. Occurrence and risk assessment of antibiotics in water and lettucein Ghana. Science of the Total Environment, Ghana, v. 622-623, p. 293305, 2018.

BELLO, M. L. Montmorilonita como excipiente farmacêutico para carreadores de fármacos: modelagem e dinâmica molecular de sistemas formados por Na-montmorinolita/fármaco. 2014. 110f. Tese (Doutorado em Ciências Farmacêuticas) - Universidade Federal do Rio de Janeiro, Rio de Janeiro, 2014.

BOTTONI, P.; CAROLI, S. Detection and quantification of residues and metabolites of medicinal products in environmental compartments, food commodities and workplaces: a review. JPBA, [S.I.], v. 106, p. 3-24, 2014.

BROWN et al, Occurrence of antibiotics in hospital, residential, and dairy effluent, municipal wastewater, and the Rio Grande. Novo México. Science of the Total Environment, [S.I.], v. 366, 772-783, 2006.

FARRÉ, M. et al, 2008. Fate and toxicity of emerging pollutants, their metabolites and transformation products in the aquatic environment. Chem, [S.I.], v. 27, p. 9911007, 2008.

GALÁN, M.J.G.; CRUZ, M.S.D.; BARCELÓ, D. Identification and determination of metabolites and degradation products of sulfonamide antibiotics. Chem, [S.I.], v. 27, p. 10081022, 2008.

GONÇALVES, A. S. Estudo da reativação da acetilcolinesterase humana inibida pelo organofosforado tabun através de métodos híbridos clássicos quanto-mecânicos. 2009. 207 f.

Tese (Doutorado em Ciências) Universidade Federal do Rio de Janeiro, Rio de Janeiro, 2009.

GONÇALVES, A. S. Estudo por dinâmica molecular das interações da pralidoxima e da deazapralidoxima com a Acetilcolinesterase humana inibida pelo agente neurotóxico Tabun. 2005. 125f. Dissertação (Mestrado em Química) - Instituto Militar de Engenharia, Rio de Janeiro, 2005.

HÖLTJE, H. D.; et al. Molecular Modeling: Basic Principles and Aplications. 2. ed. Weinheim: Wiley-VCH, 2003.

HOMEN, V. M. F., Tecnologias Alternativas de Remoção de Antibióticos de Águas Contaminadas. 2011. $341 \mathrm{f}$. Tese (Doutorado em Engenharia do Ambiente) - Faculdade de Engenharia da Universidade do Porto. Porto, Portugal, 2011.

HONÓRIO, Kathia Maria; SILVA, Alberico Borges Ferreira da. Forças intermoleculares e a interação fármacoreceptor. In: Química medicinal: métodos e fundamentos em planejamento de fármacos [S.1: s.n.], 2011.

KASSINOS, D.F., VASQUEZ, M.I., KÜMMERER, K. Transformation products of pharmaceuticals in surface waters and wastewater formed during photolysis and advanced oxidation processes -

degradation, elucidation of by products and assessment of their biological potency.

Chemosphere. [S.I.], v. 85, p. 693-709, 2011.

MATHON, B. et al. A review of the photodegradability and transformation products of 13 pharmaceuticals and pesticides relevant to sewage polishing 
treatment. Science of the Total

Environment, [S.I.], v. 551-552, p. 712-

724, 2016.

MOPAC 2016, Stewart J. J. P. MOPAC

Manual. Stewart Computational

Chemistry, Colorado Springs, USA.

Disponível em: HTTP://OpenMOPAC.

Net. Acesso em: 20. ago. 2017.

RIBEIRO, A. A. S. T.; HORTA, B. A. C.; DE ALENCASTRO, R. B. MKTOP: a program for automatic construction of molecular topologies. J. Braz. Chem. Soc, [S.I.], v.19, p. 1433-1435, 2008.

ROWLEY, C. HASSINEN T., Manual do Ghemical. Disponível em: http://www. xplora.org/downloads/Knoppix/ghemical/d oc/index.html. Acesso em: 12 de junho de 2017.

SHRIVER, D. F. et al. Química

Inorgânica. 4. ed. Porto Alegre: Bookman, 2008.

SILVA, A. W. S.; VRANKEN, Wim F. ACPYPE - AnteChamber PYthon Parser interfacE. BMC Research Notes, [S.I.], v. 5, n. 1, p. 367, 2012.

SHARMA, A.; GUPTA H. Molecular modeling. Journal of Pharmacy and Bioallied Sciences, [S.I.] vo. 1, n. 1, p. 16 $-22,2009$.

TORRES, N.H et al. Analysis and occurrence of residues of the hormones estriol, 17alpha-ethinylestradiol and 17 beta-estradiol in urban water supply by HPLCDAD. IOSRJEN Journal of Engineering. v. 2, p. 984-989, 2012.

ZHENG, Y.; ZAOUI, A. How Water and Counterions Diffuse into the Hydrated Montmorillonite. Solid State Ionics, [S.I.], v. 203, p. 80-85, 2011. 